\title{
Cavity formation in deformed amorphous solids on the nanoscale
}

\author{
Kallol Paul $\odot,{ }^{1}$ Ratul Dasgupta, ${ }^{2}$ Jürgen Horbach, ${ }^{3}$ and Smarajit Karmakar $\odot^{1, *}$ \\ ${ }^{1}$ TIFR Center for Interdisciplinary Science, Tata Institute of Fundamental Research, 36/P Gopanpally Village, \\ Serilingampally Mandal, RR District, Hyderabad 500075, Telangana, India \\ ${ }^{2}$ Department of Chemical Engineering, IIT Bombay, Powai, Mumbai 400076, India \\ ${ }^{3}$ Institute für Theoretische Physik II, Heinrich-Heine-Universität Düsseldorf, 40225 Düsseldorf, Germany
}

(Received 24 January 2020; revised 3 June 2020; accepted 1 October 2020; published 15 October 2020)

\begin{abstract}
Amorphous solids, confined on the nanoscale, are studied using molecular dynamics computer simulation. Unlike in bulk samples, failure patterns in such systems are strongly affected by the confinement geometry and interfacial effects in the sample. We study a confined model glass, subjected to uniaxial loading, for varying aspect ratios of the sample geometry. While for a small aspect ratio, the sample breaks by forming a neck, above a critical value of the aspect ratio, cavitation is seen. The critical aspect ratio is associated with a strain-rate- and temperature-dependent critical curvature of the neck, above which the free energy of the system is minimized by the formation of a cavity. This mechanism of cavity formation is probably a generic mechanism for material's failure in small confined systems under mechanical load.
\end{abstract}

DOI: 10.1103/PhysRevResearch.2.042012

Introduction. Understanding the failure mechanisms of amorphous solids under external load is one of the most active research fields in materials physics and engineering [1-14]. It is experimentally known that amorphous solids have often much larger failure strengths than their crystalline counterparts with similar compositions, but they may also show catastrophic failure, which severely limits their applicability as useful design materials [9,13,15-19]. Bulk materials that exhibit catastrophic failure by forming cracks under an applied load are generically termed as brittle materials [16,20-22], as opposed to ductile materials that show significant plastic deformation before final failure [23,24]. In the past, a lot of detailed studies have been done to understand the failure and yielding mechanisms in bulk amorphous solids [5-7,25-40], but very few studies have focused on the microscopic mechanisms of failure at the nanoscale [41-43].

Recent studies [44-49] suggest that the nature of plasticity in materials changes significantly with system size. While typically a bulk metallic glass can sustain only up to $2 \%$ strain before failing via brittle crack formation, uniaxial strain experiments on metallic glasses with different sample sizes [46] show that the strain before failure can increase up to $200 \%$ when the sample size is reduced to $100 \mathrm{~nm}$. Often, in nanosized amorphous samples, the material fails via the formation of necks. These necks can shrink to chainlike structures as thin as a few atomic layers, indicating a ductile rather than a brittle response of the material to deformation. A

\footnotetext{
*smarajit@tifrh.res.in

Published by the American Physical Society under the terms of the Creative Commons Attribution 4.0 International license. Further distribution of this work must maintain attribution to the author(s) and the published article's title, journal citation, and DOI.
}

similar observation has been also made in uniaxial compression experiments on pilers made of metallic glasses [44]. While pilers with diameters larger than $100 \mathrm{~nm}$ show prominent shear band formation, a hallmark of brittle failure, pilers that are less than $100 \mathrm{~nm}$ in diameter display homogeneous plastic deformation without shear localization. Similarly, Greer et al. [47] have demonstrated that the brittleto-ductile transition in Zr-based metallic glasses is associated with a critical sample size. Furthermore, several experiments and simulations of metallic glasses confirm that size reduction increases the material ductility due to surface states $[45,46]$. A microscopic understanding of all these observations would be very important for the systematic development of nanomaterials with specific mechanical properties.

In this article, the nature of plasticity of two- and threedimensional glasses is investigated via uniaxial elongation simulations at the nanometer length scale. In the $x$ direction, the samples are bounded by walls, while free boundaries are assumed in the $y$ direction. The systems are deformed by moving the two walls in opposite direction. We find two qualitatively different responses to the external mechanical load. On the one hand, there is necking corresponding to a ductile behavior, and on the other hand, there is the occurrence of cavities that eventually lead to a brittle failure of the sample. At a given temperature and strain rate whether the deformed systems fail via neck formation or via the formation of cavities is determined by the aspect ratio of the sample geometry. While below a critical aspect ratio the samples fail via necking, above this critical ratio the mechanical failure is due to the formation of cavities. We show that the critical aspect ratio is related to a critical curvature of the neck.

Simulation details. We have performed nonequilibrium molecular dynamics simulations of a glass-forming binary Lennard-Jones (LJ) mixture (see the Supplementary Material (SM) for further details on the model [50]). We first 


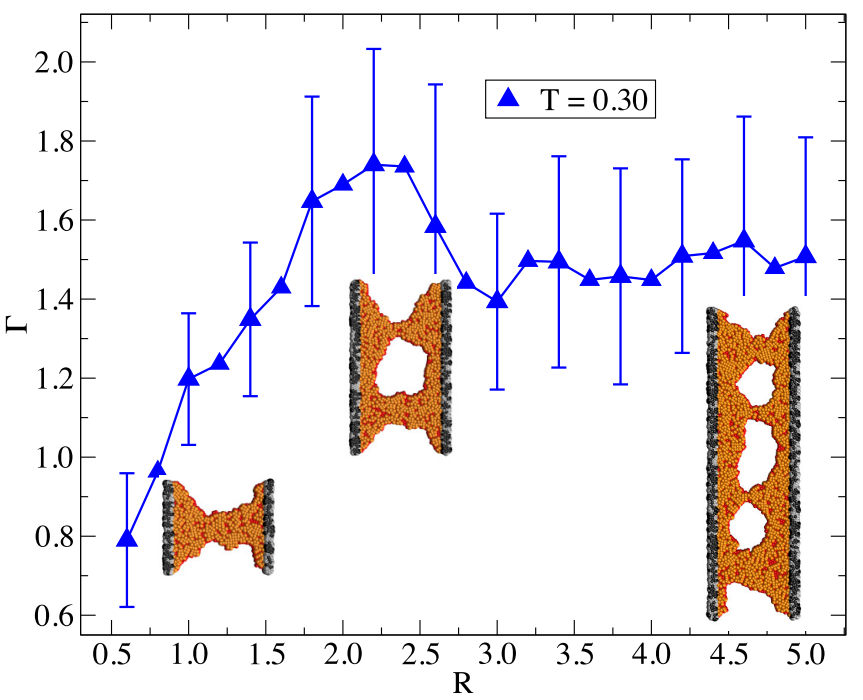

FIG. 1. Variation of maximum strain, $\Gamma$, with increasing aspect ratio, $R$, at $T=0.30$ and $\dot{\gamma}=5 \times 10^{-6}$. The crossover in failure mechanism is also shown by plotting the morphologies of the samples before the complete failure.

equilibrate the systems in the liquid state at a high temperature and then cool it to a low temperature below the calorimetric glass transition temperature $\left(T_{g} \simeq 0.33\right)$ of the model (in the following, units of length, time, and temperature are given in reduced LJ units; see SM for details). Then, at that low temperature, we couple the system to a barostat and perform constant pressure and temperature (NPT) simulations at zero pressure, followed by a removal of the periodic boundary conditions. To this end, we define two side walls at the two ends of the solid in the $x$ direction by freezing the motion of the particles in the two end regions; the typical width of the walls is around three interparticle diameters. The remaining boundaries are made free.

Next, the two walls are moved by an increment equal in size and opposite in sign; i.e., the system is subjected to uniaxial strain. To quantify the maximum deformation that a system can withstand before failure, we define the maximum strain as $\Gamma=\left(L_{f}-L_{0}\right) / L_{0}$, where $L_{0}$ is the initial length of the undeformed system along the tension direction ( $x$ direction) and $L_{f}$ is the final length just before the system breaks into two parts. We have performed simulations for different strain rates $\dot{\gamma}$, temperatures $T$, and aspect ratios $R=H / L_{0}$ (with $H$ being the lateral dimension of the walls). The strain at any instance is defined as $\gamma=\left(L-L_{0}\right) / L_{0}$, where $L$ is the length of the system at that instance. The aspect ratio $R$ is varied from 0.6 to 5.0 , keeping $L_{0}=30.0$.

Necking versus cavity formation. Figure 1 shows the maximum deformation $\Gamma$ as a function of the aspect ratio, $R$, for $T=0.30$ and $\dot{\gamma}=5 \times 10^{-6}$. As one decreases the size of the sample (i.e., the aspect ratio $R$ ), the ductility of the system significantly changes, in agreement with experimental findings [44-47]. From our data, one can infer the existence of a critical aspect ratio, $R_{c}$, below which one no longer sees an increase in maximum strain, $\Gamma$. $\Gamma$ actually starts to decrease very rapidly with decreasing aspect ratio below this critical aspect ratio, $R_{c}$. Thus, the $\Gamma$ versus $R$ curve shows a peak
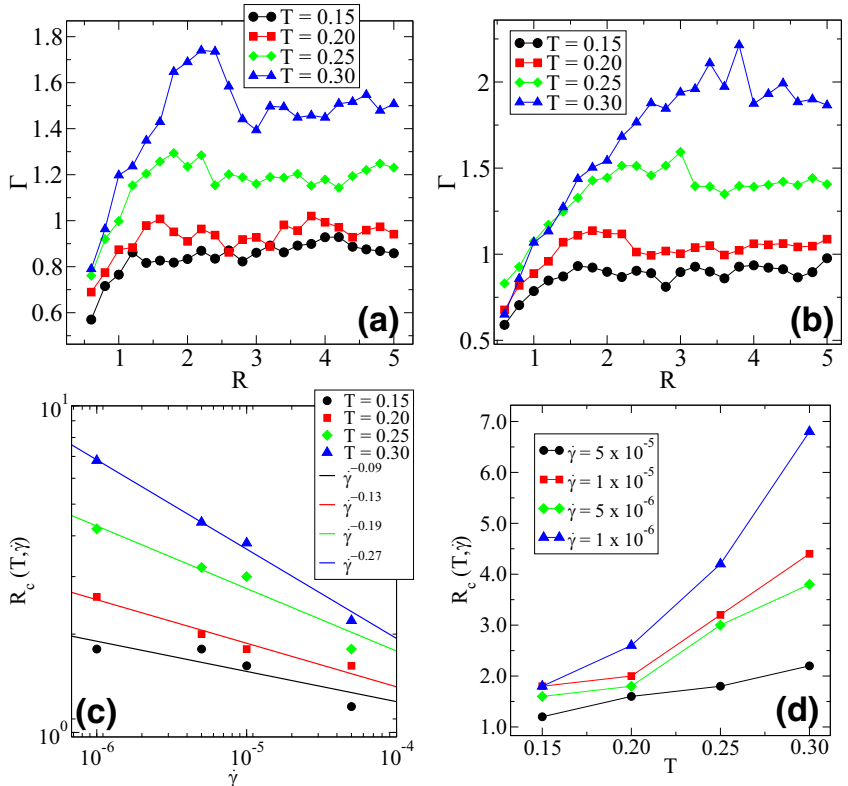

FIG. 2. Maximum strain $\Gamma$ as a function of the aspect ratio $R=$ $H / L$ for different temperature $T$ at strain rates $\dot{\gamma}=5 \times 10^{-5}$ (a) and $10^{-5}$ (b). Variation of the critical aspect ratio, $R_{c}$, as function of strain rate (c) and temperature (d).

which signifies the existence of a favored geometric aspect ratio at which the material will show maximum ductility.

Now we investigate the failure patterns as one increases the aspect ratio, in order to reveal the origin for the existence of a critical aspect ratio and a maximum ductility. As shown in Fig. 1, we found that at very small aspect ratio, $R<R_{c}$, the system always breaks via neck formation and the failure mechanism changes very sharply at the critical aspect ratio at which one observes the appearance of a single cavity. With further loading, the cavity increases in size and eventually the cavity becomes large enough that the system breaks into two fragments. As one further increases the aspect ratio, the number of cavities increases by an integer number and one observes the appearance of multiple cavities. At these higher aspect ratios, the system eventually fails by the merger of cavities into a larger cavity, in complete agreement with experimental observations [23,25,51-54] (see the SM [50] for further discussion). We believe that the appearance of cavities above a critical aspect ratio suggests a sharp ductile-to-brittle transition in the material with increasing system size.

Effects of $\dot{\gamma}$ and $T$. In Fig. 2, we show the maximum strain, $\Gamma$, as a function of the aspect ratio for different temperatures for a given strain rate. We observe that the critical aspect ratio $R_{c}$ increases with increasing temperature and the variation of $R_{c}$ with temperature is larger as one decreases the strain rate, $\dot{\gamma}$. As shown in the different panels of Fig. 2, the critical aspect ratio changes from $R_{c}=1.2$ to 2.2 for the strain rate $\dot{\gamma}=5 \times$ $10^{-5}$ [Fig. 2(a)] as the temperature increases from $T=0.15$ to 0.30 , while with slower strain rate $\dot{\gamma}=10^{-5}$, the value of $R_{c}$ increases from 1.6 to around 3.8 in the same temperature range. In Fig. 2(c), the critical aspect ratio, $R_{c}(T, \dot{\gamma})$, is plotted as a function of $\dot{\gamma}$ for different temperatures. It seems that the critical aspect ratio has a power law dependence with 


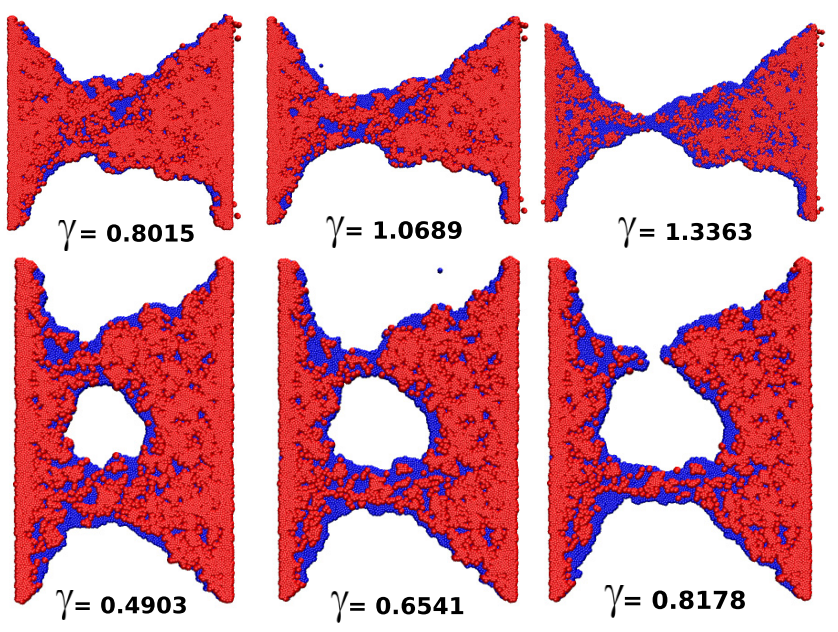

FIG. 3. Map of $D_{\min }^{2}$ as a function of strain for two aspect ratios corresponding to failure patterns of necking (top) and cavitation (bottom). Blue and red correspond to values of $D_{\min }^{2}$ that are respectively larger or lower than the average value.

the strain rate, as $R_{c}(T, \dot{\gamma}) \sim \dot{\gamma}^{\eta}$, with exponent $\eta$ varying strongly with temperature. At low temperature, $\eta$ is very small and approximately close to -0.1 and becomes substantially larger in magnitude as the temperature is increased towards $T_{g}$. At $T=0.30$, the exponent becomes $\eta \approx-0.27$. In Fig. $2(\mathrm{~d})$, the critical aspect ratio is plotted as a function of temperature for different strain rates. One clearly sees that the critical aspect ratio (at which failure happens via cavity formation) increases to larger values, indicating the expected behavior of increasing ductility with increasing temperature. The behavior becomes very pronounced with decreasing strain rate, so under experimental conditions with very slow strain rates [55], one may expect a stronger dependence on temperature.

In several studies, it has been suggested that the change from a brittle to a ductile response with decreasing system size is related to an enhanced relaxation on surface layers $[7,8,20,45,47,56]$. To characterize the surface relaxation, we have computed the nonaffine deformation of the particles quantified by $D_{\min }^{2}$ [57] and found that indeed relaxation at the surface is enhanced compared to the bulk (Fig. 3; see SM for the definition of $D_{\min }^{2}$ and further details). Blue and red in the map correspond to values of $D_{\min }^{2}$ that are respectively larger or lower than the average value. Clustering of fast particle (blue) near the open surfaces clearly suggests that relaxation is faster in the vicinity of the free surface.

Curvature of the open surface. To understand the origin of the crossover in the failure mechanism from necking to cavitation, we now analyze the curvature of the open surfaces with increasing strain. To this end, we fit the average morphology of each of the free surfaces to an ellipse and obtain the radius of curvature $\left(\mathcal{K}=a^{2} / b\right)$ at the center of the surface (vertex of the minor axis of the ellipse). Figure 4(a) shows a typical snapshot of the surface particles and a fit to it with the equation of an ellipse. As the fitting of the morphology of the open surface is observed to be very good, the estimate of the curvature is expected to be reliable.

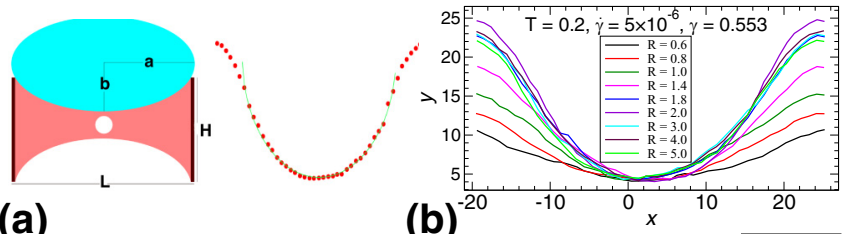

(a)

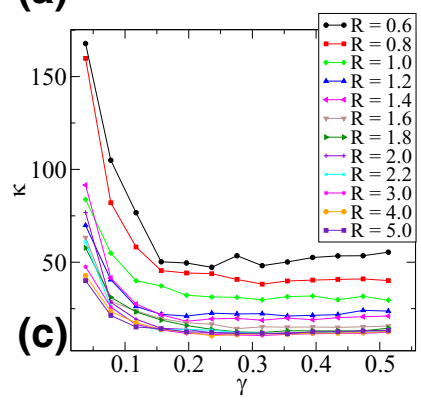

(b)
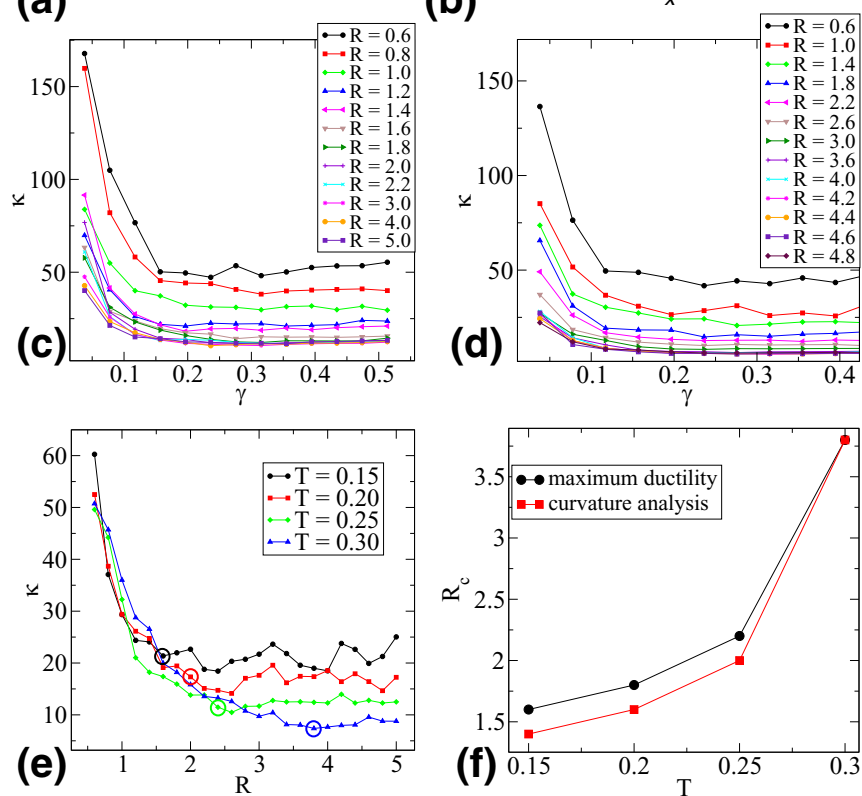

FIG. 4. (a) Schematic diagram of a surface fitting with an ellipse, (b) curvature of open surface at a constant strain for different aspect ratios, and (c) radius of curvature $\mathcal{K}$ as a function of strain for different aspect ratios $R$ at $T=0.25$ and $\dot{\gamma}=10^{-5}$. (d) The same as in panel (c) but now for $\dot{\gamma}=10^{-6}$ (e) $\mathcal{K}$ vs $R$ for different temperatures $T$ at $\dot{\gamma}=10^{-5}$. Circles in panels (e) represent the critical aspect ratio obtained from $\Gamma$ vs $R$ curves. (f) Corresponding comparisons of critical aspect ratio obtained from curvature analysis and $\Gamma$ vs $R$ curves as a function of $T$ are shown for strain rates $\dot{\gamma}=5 \times 10^{-5}$.

In Fig. 4(b), we show the curvature of the free boundary at $T=0.20$ and $\dot{\gamma}=5 \times 10^{-6}$ for different aspect ratios. It is clear that the radius of curvature $(\mathcal{K})$ is decreasing up to a certain value as the aspect ratio $R$ is increased. The radius of curvature then starts to saturate at $R \simeq 2.0$, which is close to the critical aspect ratio $R_{c}$ at $T=0.20$ and $\dot{\gamma}=5 \times 10^{-6}$. This observation indicates that once $R$ reaches $R_{c}$, the curvature of the surface can no longer increase. Thus, the system gets constrained to generate more surface area by increasing the curvature via necking and it leads to cavity formation in the bulk. Figures 4(c) and 4(d) show the evolution of curvature as a function of strain, $\gamma$, for different aspect ratios $R$ at a particular temperature $T=0.25$ and strain rates $\dot{\gamma}=5 \times 10^{-5}$ [Fig. 4(c)] and $10^{-6}$ [Fig. 4(d)]. The radius of curvature initially decreases monotonically and then reaches a plateau with increasing strain for each aspect ratio. Beyond the critical aspect ratio, the curvature saturates to the same value for all aspect ratios. The aspect ratio at which this happens tends to increase with decreasing strain as can be observed from the data shown in Figs. 4(c) and 4(d). In Fig. 4(e), we show the radius of curvature $\mathcal{K}$ at $\gamma=0.236$ as a function of aspect ratio $R$ for different temperatures $T$ and strain rate $\dot{\gamma}=10^{-5}$. For all the studied cases, the radius of curvature starts to 

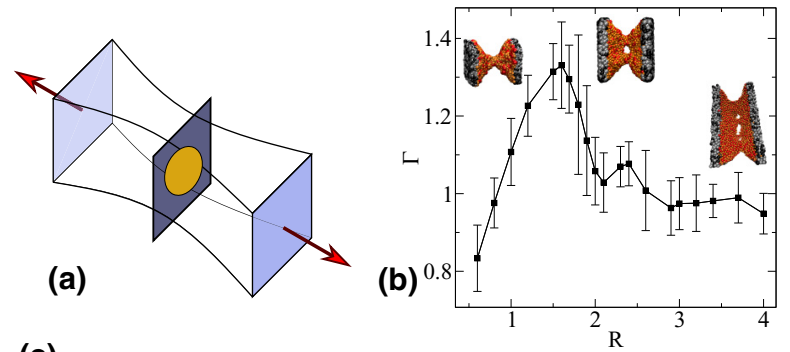

(c)
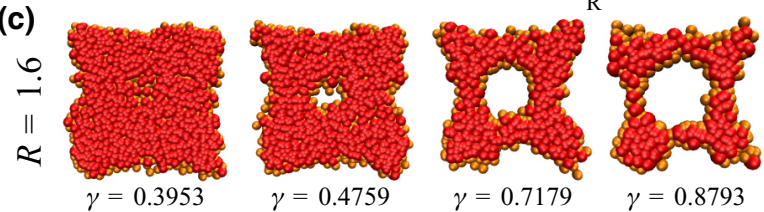

(d)
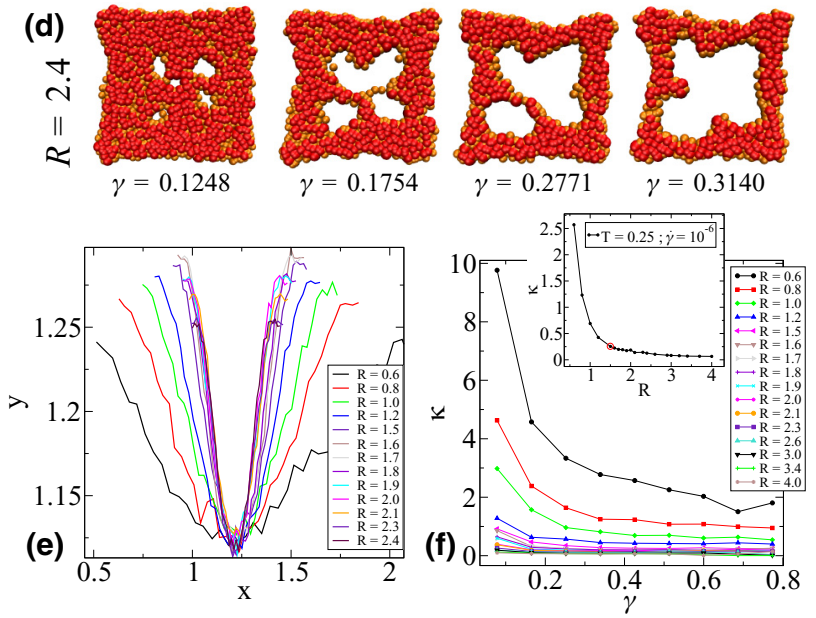

FIG. 5. Results in 3d: (a) The schematic presentation of the geometry of the uniaxial extensional simulation. The arrows represent the pulling directions and the cross section shows the middle plane where the cavity analyses are done. (b) The maximum strain, $\Gamma$, as a function of the aspect ratio. (c) Snapshots of single cavity formation. (d) Snapshots that show the formation of multiple cavities. (e) Variation of free surface profile for different aspect ratios $R$. (f) Variation of the curvature of open surface as a function of strain for different $R$. Inset: The change in $\mathcal{K}$ as a function of $R$ for $T=0.25$ and $\dot{\gamma}=10^{-6}$. The red circle shows the critical aspect ratio, as obtained from the $\Gamma$ vs $R$ curve.

saturate after values of $R$ that are close to the critical aspect ratio, $R_{c}$, at that $T$ and $\dot{\gamma}$. In Fig. 4(f), we compare the value of the $R_{c}$ computed from curvature analysis with the one computed earlier from the variation of $\Gamma$ with $R$. The correspondence is indeed very good (see the SM [50] for details).

Results in three dimensions. In three dimensions (3d), a larger variety of different confined geometries than in $2 \mathrm{~d}$ is possible. Here, we consider a bar geometry in which the length along the elongation axis is chosen as $L_{0}$ with a quadratic cross section of the bar of area $H \times H$ [cf. Fig. 5(a)]. Similarly to the $2 \mathrm{~d}$ case, the aspect ratio is defined as $R=$ $H / L_{0}$ and we investigate the behavior of the maximum strain $\Gamma$ as a function of $R$. In Fig. 5(b), we show $\Gamma$ versus. $R$ at $T=0.20$ and strain rate $\dot{\gamma}=1 \times 10^{-5}$. With increasing $R, \Gamma$ reaches a peak at a critical aspect ratio, $R_{c}$, and then decreases quite sharply with further increase in aspect ratio. Note the undulation in $\Gamma$ versus $R$ curve after the first peak. This seems to be related to stepwise increase in number of cavities around that particular aspect ratio (see the SM [50] for a detailed discussion). The results are very similar to the ones obtained for the $2 \mathrm{~d}$ systems. While for $R<R_{c}$, necking is observed, for $R>R_{c}$, cavities form. Again, for large aspect ratios, multiple cavities are seen, followed by a subsequent merger before the final failure. Thus, the existence of a critical aspect ratio above which cavitation dominates the failure mechanisms under uniaxial elongation tests seems to be very generic and does not depend on the dimensionality.

Next, we do a similar curvature analysis of the open surfaces as in the $2 \mathrm{~d}$ case to see whether similar mechanisms hold also in 3d. Figures 5(c) and 5(d) display snapshots of cavity formation at different strains for the aspect ratios $R=1.6$ and $R=2.4$, respectively. These snapshots show the middle plane between the two walls where, due to the symmetry, the first nucleation of a cavity is expected. In Fig. 5(c), i.e., for $R=1.6$ just after the critical aspect ratio, a single cavity forms, whereas for $R=2.4$ multiple cavities are expected; cf. Fig. 5(d). As there are four equivalent open surfaces, multiple cavities also appear in a symmetrical manner. Therefore, one obtains first a single cavity and then four cavities with increasing aspect ratios in accordance with the quadratic cross section of the bar. This suggests that one can tune the cavity formation and their patterns by choosing an appropriate aspect ratio in combination with the cross-sectional geometry of the sample, indicating that the failure mechanism in a $3 \mathrm{~d}$ nanosized sample can be tuned, too. The systematic variation of geometrical shapes will be an interesting issue for forthcoming studies.

Figures 5(e) and 5(f) show the results of our curvature analysis. For this, we have taken a cross-sectional cut along the pulling axis and computed the radius of curvature for varying aspect ratio and strain. As the sample has symmetry perpendicular to the pulling axis, a cross-sectional cut along the pulling axis will capture the variation of the curvature of the open surface without any ambiguity. In Fig. 5(e), the shape of the open surface is shown at a fixed strain $(\gamma=0.5163)$ for different aspect ratios. One clearly sees that with increasing $R$, the shape of the open surface becomes independent of $R$. In Fig. 5(f), the radius of curvature, $\mathcal{K}$ is shown as a function of strain for different $R$. In the inset of Fig. 5(f), the radius of curvature, $\mathcal{K}$, at large strain, is plotted as a function of $R$. The red circle shows the critical aspect ratio $R_{c}$ obtained from the $\Gamma$ versus $R$ plots. The value of $R_{c}$ is very close to the aspect ratio at which the large strain radius of curvature also saturates as a function of $R$. These analyses indicate that for the bar geometry the failure mechanisms at the nanoscale is very similar to that for the $2 \mathrm{~d}$ system.

Summary and conclusions. We have performed uniaxial extensile deformation simulations of confined two- and threedimensional model glasses at different temperatures, $T$, strain rates, $\dot{\gamma}$, and aspect ratios of the system's geometry, $R$. For sufficiently low values of $R$, the systems fail via necking. For $R>R_{c}$, however, failure is associated with the occurrence of one or more cavities. Cavities form because there is a maximum strain-rate-dependent radius of curvature of the neck, $\mathcal{K}_{\max }$. The formation of cavities provides an effective minimization of a nonequilibrium free energy of the system in the presence of a finite strain rate. The change in the failure mechanism around a critical aspect ratio $R_{c}$ resembles 
the ductile-to-brittle transition in bulk systems, with cavities leading to a brittle-like response of the confined glass systems. Thereby, we have revealed an interesting mechanism of failure in small confined systems under mechanical load.

Acknowledgments. We would like to thank Pinaki Chaudhuri, Surajit Sengupta, and Srikanth Sastry for useful discus- sions. This project is funded by intramural funds at TIFR Hyderabad from the Department of Atomic Energy (DAE). K.P. wants to thank SB/SFJ/2019-20/05 for financial support. S.K. would like to acknowledge the support from Swarna Jayanti Fellowship Grants No. DST/SJF/PSA-01/2018-19 and No. SB/SFJ/2019-20/05
[1] A. S Argon, Plastic deformation in metallic glasses, Acta Metall. 27, 47 (1979).

[2] A. L. Greer, Metallic glasses, Science 267, 1947 (1995).

[3] C. E. Maloney and A. Lemaître, Amorphous systems in athermal, quasistatic shear, Phys. Rev. E 74, 016118 (2006).

[4] H. G. E. Hentschel, S. Karmakar, E. Lerner, and I. Procaccia, Size of Plastic Events in Strained Amorphous Solids at Finite Temperatures, Phys. Rev. Lett. 104, 025501 (2010).

[5] S. Karmakar, E. Lerner, I. Procaccia, and J. Zylberg, Statistical physics of elastoplastic steady states in amorphous solids: Finite temperatures and strain rates, Phys. Rev. E 82, 031301 (2010).

[6] S. Karmakar, E. Lerner, and I. Procaccia, Statistical physics of the yielding transition in amorphous solids, Phys. Rev. E 82, 055103(R) (2010).

[7] O. Dauchot, S. Karmakar, I. Procaccia, and J. Zylberg, Athermal brittle-to-ductile transition in amorphous solids, Phys. Rev. E 84, 046105 (2011).

[8] M. Lagos and R. Das, Brittle and ductile character of amorphous solids, Adv. Appl. Math. Mech. 8, 485 (2016).

[9] T. C. Hufnagel, C. A. Schuh, and M. L. Falk, Deformation of metallic glasses: Recent developments in theory, simulations, and experiments, Acta Mater. 109, 375 (2016).

[10] M. Chen, Mechanical behavior of metallic glasses: Microscopic understanding of strength and ductility, Annu. Rev. Mater. Res. 38, 445 (2008).

[11] M. F. Ashby and A. L. Greer, Metallic glasses as structural materials, Scr. Mater. 54, 321 (2006), viewpoint set no. 37: On mechanical behavior of metallic glasses.

[12] D. Rodney, A. Tanguy, and D. Vandembroucq, Modeling the mechanics of amorphous solids at different length scale and time scale, Modell. Simul. Mater. Sci. Eng. 19, 083001 (2011).

[13] C. Schuh, T. Hufnagel, and U. Ramamurty, Overview no. 144: Mechanical behavior of amorphous alloys, Acta Mater. 55, 4067 (2007).

[14] G. P. Shrivastav, P. Chaudhuri, and J. Horbach, Yielding of glass under shear: A directed percolation transition precedes shearband formation, Phys. Rev. E 94, 042605 (2016).

[15] K. Binder, J. Horbach, W. Kob, and A. Winkler, Computer simulation of molten and glassy silica and its mixtures with sodium oxide and aluminium oxide, in Complex Inorganic Solids: Structural, Stability, and Magnetic Properties of Alloys (Springer, Boston, 2005), pp. 35-53.

[16] J. Schroers and W. L. Johnson, Ductile Bulk Metallic Glass, Phys. Rev. Lett. 93, 255506 (2004).

[17] B. E. Schuster, Q. Wei, M. H. Ervin, S. O. Hruszkewycz, M. K. Miller, T. C. Hufnagel, and K. T. Ramesh, Bulk and microscale compressive properties of a Pd-based metallic glass, Scr. Mater. 57, 517 (2007).
[18] S. Scudino, H. Shakur Shahabi, M. Stoica, I. Kaban, B. Escher, U. Kühn, G. B. M. Vaughan, and J. Eckert, Structural features of plastic deformation in bulk metallic glasses, Appl. Phys. Lett. 106, 031903 (2015).

[19] B. A. Sun and W. H. Wang, The fracture of bulk metallic glasses, Prog. Mater. Sci. 74, 211 (2015).

[20] H. Guo, P. F. Yan, Y. B. Wang, J. Tan, Z. F. Zhang, M. L. Sui, and E. Ma, Tensile ductility and necking of metallic glass, Nat. Mater. 6, 735 (2007).

[21] J. P. Crété, P. Longère, and J. M. Cadou, Numerical modelling of crack propagation in ductile materials combining the GTN model and X-FEM, Comput. Methods Appl. Mech. Eng. 275, 204 (2014)

[22] G. C. Li, H. Q. Liu, M. L. Du, Y. S. Hong, and X. Zhang, Crack tip behaviour and crack propagation in ductile materials, Fatigue Fract. Eng. Mater. Struct. 15, 187 (1992).

[23] I. Singh, R. Narasimhan, and U. Ramamurty, CavitationInduced Fracture Causes Nanocorrugations in Brittle Metallic Glasses, Phys. Rev. Lett. 117, 044302 (2016).

[24] P. Chaudhuri and J. Horbach, Structural inhomogeneities in glasses via cavitation, Phys. Rev. B 94, 094203 (2016).

[25] P. Guan, S. Lu, M. J. B. Spector, P. K. Valavala, and M. L. Falk, Cavitation in Amorphous Solids, Phys. Rev. Lett. 110, 185502 (2013).

[26] K. Singh and Y. Rabin, Effect of Liquid State Organization on Nanostructure and Strength of Model Multicomponent Solids, Phys. Rev. Lett. 123, 035502 (2019).

[27] C. H. Rycroft and E. Bouchbinder, Fracture Toughness of Metallic Glasses: Annealing-Induced Embrittlement, Phys. Rev. Lett. 109, 194301 (2012).

[28] M. Vasoya, C. H. Rycroft, and E. Bouchbinder, Notch Fracture Toughness of Glasses: Dependence on Rate, Age, and Geometry, Phys. Rev. Appl. 6, 024008 (2016).

[29] E. Irani, P. Chaudhuri, and C. Heussinger, Athermal rheology of weakly attractive soft particles, Phys. Rev. E 94, 052608 (2016).

[30] J. Rottler and M. O. Robbins, Shear yielding of amorphous glassy solids: Effect of temperature and strain rate, Phys. Rev. E 68, 011507 (2003).

[31] J. Rottler and M. O. Robbins, Growth, microstructure, and failure of crazes in glassy polymers, Phys. Rev. E 68, 011801 (2003).

[32] J. Rottler and M. O. Robbins, Yield conditions for deformation of amorphous polymer glasses, Phys. Rev. E 64, 051801 (2001).

[33] F. Varnik, L. Bocquet, and J.-L. Barrat, A study of the static yield stress in a binary Lennard-Jones glass, J. Chem. Phys. 120, 2788 (2004).

[34] K. Martens, L. Bocquet, and J.-L. Barrat, Connecting Diffusion and Dynamical Heterogeneities in Actively Deformed Amorphous Systems, Phys. Rev. Lett. 106, 156001 (2011). 
[35] A. Nicolas, E. E. Ferrero, K. Martens, and J.-L. Barrat, Deformation and flow of amorphous solids: Insights from elastoplastic models, Rev. Mod. Phys. 90, 045006 (2018).

[36] B. P. Bhowmik, P. Chaudhuri, and S. Karmakar, Effect of Pinning on the Yielding Transition of Amorphous Solids, Phys. Rev. Lett. 123, 185501 (2019).

[37] P. Nath, S. Ganguly, J. Horbach, P. Sollich, S. Karmakar, and S. Sengupta, On the existence of thermodynamically stable rigid solids, Proc. Natl. Acad. Sci. USA 115, E4322 (2018).

[38] R. Dasgupta, S. Karmakar, and I. Procaccia, Universality of the Plastic Instability in Strained Amorphous Solids, Phys. Rev. Lett. 108, 075701 (2012).

[39] P. Leishangthem, A. D. S. Parmar, and S. Sastry, The yielding transition in amorphous solids under oscillatory shear deformation, Nat. Commun. 8, 14653 (2017).

[40] M. Ozawa, L. Berthier, G. Biroli, A. Rosso, and G. Tarjus, Random critical point separates brittle and ductile yielding transitions in amorphous materials, Proc. Natl. Acad. Sci. USA 115, 6656 (2018).

[41] A. R. C. Baljon and M. O. Robbins, Energy dissipation during rupture of adhesive bonds, Science 271, 482 (1996).

[42] A. R. C. Baljon and M. O. Robbins, Simulations of crazing in polymer glasses: Effect of chain length and surface tension, Macromolecules 34, 4200 (2001).

[43] C. D. Lorenz and M. J. Stevens, Fracture behavior of LennardJones glasses, Phys. Rev. E 68, 021802 (2003).

[44] C. A. Volkert, A. Donohue, and F. Spaepen, Effect of sample size on deformation in amorphous metals, J. Appl. Phys. 103, 083539 (2008).

[45] D. Z. Chen, D. Jang, K. M. Guan, Q. An, W. A. Goddard, and J. R. Greer, Nanometallic glasses: Size reduction brings ductility, surface state drives its extent, Nano Lett. 13, 4462 (2013).

[46] J. H. Luo, F. F. Wu, J. Y. Huang, J. Q. Wang, and S. X. Mao, Superelongation and Atomic Chain Formation in Nanosized Metallic Glass, Phys. Rev. Lett. 104, 215503 (2010).
[47] D. Jang and J. R. Greer, Transition from a strong-yet-brittle to a stronger-and-ductile state by size reduction of metallic glasses, Nat. Mater. 9, 215 (2010).

[48] J. R. Greer and J. T. M. De Hosson, Plasticity in small-sized metallic systems: Intrinsic versus extrinsic size effect, Progr. Mater. Sci. 56, 654 (2011).

[49] O. Kraft, P. A. Gruber, R. Mönig, and D. Weygand, Plasticity in confined dimensions, Annu. Rev. Mater. Res. 40, 293 (2010).

[50] See Supplemental Material at http://link.aps.org/supplemental/ 10.1103/PhysRevResearch.2.042012 for more details on models and methods and other supporting analyses that are referred to in this Rapid Communication.

[51] B. Ding, X. Li, X. Zhang, H. Wu, Z. Xu, and H. Gao, Brittle versus ductile fracture mechanism transition in amorphous lithiated silicon: From intrinsic nanoscale cavitation to shear banding, Nano Energy 18, 89 (2015).

[52] R. Maaß, P. Birckigt, C. Borchers, K. Samwer, and C. A. Volkert, Long range stress fields and cavitation along a shear band in a metallic glass: The local origin of fracture, Acta Mater. 98, 94 (2015).

[53] Q. An, G. Garrett, K. Samwer, Y. Liu, S. V. Zybin, S.-N. Luo, M. D. Demetriou, W. L. Johnson, and W. A. Goddard, Atomistic characterization of stochastic cavitation of a binary metallic liquid under negative pressure, J. Phys. Chem. Lett. 2, 1320 (2011).

[54] P. Murali, T. F. Guo, Y. W. Zhang, R. Narasimhan, Y. Li, and H. J. Gao, Atomic Scale Fluctuations Govern Brittle Fracture and Cavitation Behavior in Metallic Glasses, Phys. Rev. Lett. 107, 215501 (2011)

[55] A. V. Sergueeva, N. A. Mara, D. J. Branagan, and A. K. Mukherjee, Strain rate effect on metallic glass ductility, Scr. Mater. 50, 1303 (2004).

[56] P. S. Steif, Ductile versus brittle behavior of amorphous metals, J. Mech. Phys. Solids 31, 359 (1983).

[57] M. L. Falk and J. S. Langer, Dynamics of viscoplastic deformation in amorphous solids, Phys. Rev. E 57, 7192 (1998). 